müssen auch die in den vorstehenden Untersuchungen berührten Wirkungen der Haloide usw. als Umhüllungserscheinungen aufgefaßt werden, die verständlicherweise eine um so gröBere Rolle spielen müssen, je größier die spezifische Oberflache, also je höher der Dispersitätsgrad des Silbers ist. Wir sahen diese Wirkung von Schutzhüllen zunächst bei der Vèthinderung der Agglutination der kleinsten gelbroten Silbersolteilchen zu grünen, ferner bei dem starken Einflub auf die Struktur des Silbergels, das mit steigendem ursprünglichen Dispersitătsgrade in Gegenwatt von Haloid oder Zyanid bei der Reduktion reaktionsfähiger gegenüber Quecksilberchlorid wird. Bei jenen Gelen laßt sich zeigen, daß sie die ganze bei der Reduktion zugefügte Chlormenge als Chlorsilber im Niederschlage enthalten. Die die einzelnen Aggregate trennenden Schutzhillen bestehen demnach aus Chlorsilber. Dies dürtte also auch in den Gelatinesolen der Fall sein, zumal die Wirkung gegen die Agglutination der kleinsten Teilchen in genau gleicher Weise beobachtet wird, ob man die Haloidsalze schon vor der Reduktion anwendet, wobei sich ja selbstverstandlich Chlorsilber bildet, oder ob man das Haloid oder auch Zyanid erst während oder sogar erst nach der Reduktion wirken läBt.

DaB Schutzhüllen von Halogensilber in der besprochenen Art schon aus der bloben Einwirkung auch ron Neutralsalzlosungen auf kolloides Silber entstehen können und tatsächlich entstehen, ist durch die vorzüglichen Untersuchungen von P. P. v. We imar $n^{24}$ ) nachgewiesen worden. Dieser Forscher sagt $u$. a.:

"Aus all dem Dargelegten geht also vollkommen deutlich hervor, daB metallisches.Silber, das sich im Zustande einer auBerst feinen Zerteilung befindet, solche Reaktionen eingehen kann, die beim grobzerteilten Silber entweder praktisch gar nicht oder doch nur mit auBerordentlicher Langsamkeit vor sich gehen. ...

M) Koll.-Zeitschr. 5, 64 (1909).
Salze der gutlöslichen Basen ( $\mathrm{KOH}, \mathrm{NaOH}$, $\mathrm{Ba}(\mathrm{OH})_{2}$ usw.) und Säuren, die mit dem Silber schwerlosliche Silberverbindungen liefern (z. B. chlor-, brom-, jod-, rhodan-, chromsaures Silber), reagieren mit dem Silbersol unter Ausscheidung von freiem Alkali. . . . E Eine Beachtung verdienen die Koagulate der Silbersole, die durch Einwirkungen gutlöslicher Haloidsalze auf die Sole erhalten werden. Die Struktur dieser Koagulate ist der Struktur der sogen. Photohaloide entgegengesetzt, die Zusammensetzung aber identisch. ... Lüppo-Cramer lieferte vor kurzem ${ }^{25}$ ) eine einfache Darstellungsinethode der Photohaloide durch Reduktion des $\mathrm{AgNO}_{3}$ in Gegenwart 2. B. von $\mathrm{AgBr}$ nach der Methode von Carey Lea. Diese Versuche haben meiner Ansicht nach nicht nur die Anschauungen von Lüp po-Cramer bestätigt, daß die Photohaloide normale Haloide sind, die durch, kolloides* Silber gefärbt sind, sie haben auch bewiesen, das die Photohaloide nicht ,besondere Kolloidverbindungen' sind, sondern ein einfaches feines Gemisch zweier bestimmter Verbindungen der feinsten Kristallchen des Haloidsilbers und des metallischen Silbers."

Die oben beschriebene Umwandiung der katalytischen Wirkung des Silbers auf die $\mathrm{H}_{2} \mathrm{O}_{2}$ Zersetzung durch Emulsoide in eine rein chemische Reaktion - die A ufl os ung des Silbers durch das Peroxyd - legt den Gedanken nahe, $\mathrm{daB}$ unter den angegebenen Bedingungen vorübergehend kolloider Sauerst off entstanden sein könnte. Ein analoges Ausbleiben der Gasentwicklung wurde bei der katalytischen Wirkung von Eisen- und Kupferchlorid auf die $\mathrm{H}_{2} \mathrm{O}_{2}$-Zersetzung innerhalb einer Gelatinegallerte von R. Ed. Liese gang ${ }^{26}$ ) beobachtet. Auch hier ist die Hypothese nicht fernliegend, daB innerhalb des Schutzkolloides der entstehende Sauerstoff kolloid gelost sein und damit die verflüssigende Wirkung auf die Gelatine ausüben könnte.

25) Koll.-Zeitschr. 3, 360 (1908).

*) R. Ed.Liese gan g, Beiträge zu einer Kolloldchemie des Lebens (Dresden 1909), 63.

\title{
Zur Theorie der Vulkanisation des Kautschuks.
}

Von F. Willy Hinrichsen.

(Eingegangen an 17. Mărz 1911)

Die Frage nach der Natur der bei der Vulkanisation des Kautschuks sich abspielenden Vorgänge ist noch immer nicht völlig geklärt, trotzdem gerade in der jüngsten Zeit zahlreiche
Veröffentlichungen über diesen Gegenstand erschienen sind. Wärend $\mathrm{C} O$. Weber ${ }^{1}$ ) die

1) Zeitschr. $t$ angew. Chem. 1894, 112, 142. 
Aufnahme des Schwefels und Schwefelchlorürs durch den Kautschuk als rein chemische Reaktion ansprach, hat zuerst Wo. Ostwald ${ }^{2}$ ) in einer interessanten umfassenden Studie die gesamten Erscheinungen der Schwefel- und Schwefelchlorüraufnahme des Kautschuks einheitlich von dem Gesichtspunkte aus zusammenzufassen gesucht, daß es sich hierbei lediglich um Adsorptionserscheinungen handele.

Die Abhandlung von Wo. Ostwald gab F. W. Hinrichsen und E. Kindsche $r^{3}$ ) Veranlassung, in einer vorläufigen Mitteilung über eigene Versuche $z u$ berichten, die ebenfalls die Prüfung speziell der Kaltvulkanisation vom Standpunkte der Adsorptionstheorie bezweckten. Es gelang ihnen, einerseits die Adsorption von Schwefel, der aus dem Schwefelchlorür durch Feuchtigkeit abgeschieden wird, mit Sicherheit nachzuweisen, andererseits bei der Nachprüfung der Versuche $\mathrm{C}$. $\mathrm{O}$. Weber's durch Einwirkung von Schwefelchlorür auf Kautschuk in benzolischer Lösung die Bildung einer bestimmten Verbindung wahrscheinlich zu machen.

Unmittelbar nach der Abhandlung von F.W. Hinrichsen und $E$. Kindscher veröffentlichte B. Bys ow ${ }^{4}$ mehrere Arbeiten über die gleiche Frage. Bei seinen Versuchen bediente er sich jedoch eines grundsätzlich anderen Verfahrens, als es $C$. $O$. Weber und später $F$. W. Hinrichsen und E. Kindscher angewandt hatten. In Anlehnung an das bei der technischen Kaltvulkanisation übliche Vorgehen tauchte er nämlich abgewogene Stücke festen Parakautschuks in verdönnte Lösungen von Schwefelchlorür und konnte zeigen, dab die Schwefelchlorür- und bei entsprechenden Versuchen auch die Schwefelaufnahme durch den Kautschuk streng nach dem Adsorptionsgesetze $\frac{x}{a}=k \cdot c^{p}$ verlief. Spätere Untersuchungen ${ }^{5}$ ), bei denen sowohl der Schwefel- wie der Chlorgehalt der mit Schwefelchlorürlösung behandelten Proben ermittelt wurden, ergaben ferner, daß bei Anwendung von technischem, etwas feuchtem Kautschuk stets etwas mehr Schwefel als Chlor aufgenommen war, was in Uebereinstimmung mit den von F. W. Hinrichsen und $E$. Kindscher ausgeführten, allerdings von B. Bysow nicht erwähnten Versuchen

\footnotetext{
2) Koll.-Zeitschr. 6, 136 (1910).

s) Koll.-Zeitschr. 6, 202 (1910).

7) Koll.-Zeitschr. 6, 281 (1910); 7, 160 (1910)

5) Koll-Zeitschr. 8, 47 (1911).
}

durch Adsorption von Schwefel gedeutet werden kann, der unter dem Einflusse der Feuchtigkeit aus Schwefelchlorür abgeschieden wurde. B. Bys ow selbst gibt für seinen Befund keine Erklärung.

Die Arbeiten von B. Bysow sowie von F. W. Hinrichsen und E. Kindscher über Kaltvulkanisation stehen insofern in einem gewissen scheinbaren Gegensatz, als nach ersterem die Vulkanisation lediglich auf Adsorptionsvorgänge zurückzuführen wäre, während nach letzteren auch die Bildung einer bestimmten chemischen Verbindung neben Adsorption in Frage käme. Diesen Widerspruch hat Wo. Ostwald ${ }^{6}$ ) unter Berücksichtigung der in den erwähnten Arbeiten benutzten Konzentrationsverhältnisse der Schwefelchlorïrlösungen in der Weise zu beheben versucht, daß er die Ergebnisse beider Arbeiten in einer einzigen Adsorptionskurve, die die Abhängigkeit der aufgenommenen von der angewandten Schwefelchlorürkonzentration wiedergibt, darstellte. Da die typische Adsorptionskurve anfänglich stark. nach einiger Zeit schwächer ansteigt und schlieklich parallel zur Abszissenachse verläuft, können die erwähnten Ergebnisse in folgender Weise ihre Deutung finden: Die von B. Bysow bei sehr verdünnten Lösungen beobachtere starke Zunahme des Verbrauchs mit steigender Konzentration entspricht dem ersten Teile, die von F. W. Hinrichsen und E. Kindscher aufgefundene anscheinende Konstanz des Verbrauchs bei konzentrierten Lösungen, unabhängig von der Konzentration, dem letzten, asymptotisch verlaufenden Teile der Adsorptionskurve.

So überzeugend nun aber auch der Erklärungsversuch Wo. Ostwald's erscheint und wenn auch sicherlich bei der Schwefel- und Schwefelchlorïraufnahme des Kautschuks Adsorptionsvorgänge eine wesentliche Rolle spiclen, so scheint mir doch die Annahme, dab a us schlieblich Adsorptionserscheinungen für die Vulkanisation matigebend sind, nicht ausreichend $z u$ sein. Eine Reihe von Gründen. die gegen diese Annahme anzuführen sind, habe ich vor kurzem an anderer Stelle zusammengefaBt ${ }^{7}$ ). Ich kann mich daher hier auf die Hervorhebung der wichtigsten beschrünken, um danı noch einige weitere Folgerungen anzuschliehen.

Der wichtigste Einwand, der sich gegen die rein physikalische Deutung der Vulkani-

6) Koll,-Zeitschr. 7, 45 (1910).

7) "Kunststoffe" 1, 41 (1911). 
sationsvorgänge erheben läßt, ist schon vor einiger Zeit von $P$. Alexande ${ }^{8}$ ) erwähnt worden: die Schwierigkeit, die Reaktion der Schwefelaufnahme wieder rückgängig zu machen. In der Tat handelt es sich ja, wie besonders aus den eingehenden Untersuchungen $H$. Freund lich's ${ }^{9}$ ) hervorgeht, bei der Adsorption um ein Gleichgewicht, das seiner Definition nach von beiden Seiten her erreichbar sein muB. Handelt es sich beispielsweise um eine Adsorption aus einer Lösung, so muß man erwarten, daß bei nachträglicher Behandlung mit dem reinen Lösungsmittel der adsorbierende Stoff vollkommen von dem adsorbierten Bestandteil wieder zu trennen sein mub. Dies ist jedoch bei dem vulkanisierten Kautschuk keineswegs der Fall. Bekanntlich gelingt es nicht, durch bloße Behandlung mit einem beliebigen Lösungsmittel für Schwefel den bei der Vulkanisation aufgenommenen Schwefel vollständig wieder $z u$ entfernen, ja, die Entschwefelung (Devulkanisierung) des vulkanisierten Kautschuks erfolgt so schwierig, daB die Mehrzahl der praktischen Kautschukchemiker sich seit langem daran gewöhnt hat, die Devulkanisierung, d. h. die Ueberführung des vulkanisierten Kautschuks in eine dem Rohkautschuk entsprechende Form, geradezu als unlösbares Problem anzusehen. Ich möchte mich dieser letzteren Ansicht zwar nicht anschlieben, haite vielmehr auch die völlige Entschwefelung des vulkanisierten Kautschuks für sehr wohl denkbar ${ }^{10}$ ), wohl aber bin ich überzeugt, daB dieser Vorgang nicht auf rein physikalischem Wege durch einfache Herauslösung des Schwefels mit einem geeigneten Lösungsmittel, sondern höchstens auf $\mathrm{ch}$ e mischem Wege durchführbar sein dürfte.

Man hat gegen den zuerst von P. Alexander erhobenen Einwand der Nichtumkehrbarkeit der Reaktion zwischen Schwefel und Kautschuk erwidert, daß es auch unzweifelhafte Adsorptionsvorgänge gebe, die nicht umkehrbar seien, und sich hierbei vornehmlich auf gewisse Beobachtungen von $\mathrm{H}$. Freundlich und $G$. Losev" ") berufen. Wie mir scheint, mit Unrecht. Denn bei den erwähnten Untersuchungen handelt es sich nur um eine scheinbare Nichtumkehrbarkeit. H. Freundlich und G. Losev untersuchten die Adsorption von gelösten basischen Farbstoffen, z. B. von Kristallviolett, durch Kohle. Hierbei fanden cie, daB der nach Ein-

8) Chem.-Ztg. 34, 789 (1910).

9) Kapillarchemie" (Leipzig 1909).

19) Vgl. z. B. Esch, Chem.-Ztg. 34, 1082 (1910).

1) Zeitschr. A. physik. Chem. 59, 284 (1907). stellung des Gleichgewichtes auf der Oberflache der Kohle adsorbierte Farbstoff im Gegensatz zu der ursprünglich angewandten Substanz in Wasser nicht mehr löslich war. Als Grund dieser Erscheinung lieB sich aber feststellen, dab hier nicht nur eine einfache Adsorption, sondern gleichzeitig auch eine chemische Veränderung eingetreten war. Das im Kristallviolett vorliegende Salz hatte nämlich in der Lösung die gesamte in ihm enthaltene Säure (Salzsäure) an das Wasser abgegeben, und nur die Farbbase war durch die Kohle adsorbiert worden. Da die Base selbst in reinem Wasser nicht löslich war, konnte sie naturgemäB auf diesem Wege auch nicht von der Kohle entfernt werden. Ersetzte man aber das Wasser durch ein geeignetes organisches Lösungsmittel oder durch Salzsäure, so ging die Farbbase augenblicklich wieder entsprechend in Loosung. Das Adsorptionsgleichgewicht war demnach tatsächlich umkehrbar.

Bei der Aufnahme von z. B. Schwefel durch Kautschuk ist ein ähnlicher Vorgang, wie er im Falle des Kristallvioletts beobachtet worden ist, nicht ohne weiteres wahrscheinlich. Man könnte zwar, entsprechend einer Anregung von E. Heyn, an die Möglichkeit verschiedener Modifikationen des Schwefels denken, die sich dann auch in ihren Löslichkeitsverhältnissen unterscheiden würden (plastischer, rhombischer und monokliner Schwefel), jedoch sind diese Umwandlungen ja meist an bestimmte höhere Temperaturen gebunden, während die Schwefelaufnahme des Kautschuks, wie weiter unten noch besprochen werden soll, bei beliebiger Temperatur erfolgen kann ${ }^{12}$ ).

Aber selbst wenn es gelingen sollte, die Löslichkeitsunterschiede, die in der Kautschukanalyse zur Unterscheidung des azetonlöslichen "freien" und des azetonunlöslichen "gebundenen "Schwefels geführt haben, etwa durch die Auffindung bestimmter Schwefelmodifikationen $z u$ deuten, so bliebe immer noch die rein physikalisch nicht erklärbare Tatsache, dab der gebundene Schwefel quantitativ in Derivate des Kautschuks überzuge he $n$ vermag, ein Punkt, auf den ebenfalls $P$. Alexander bereits aufmerksam gemacht hat. Da diese Derivate des Kautschuks zum Teil aus Lösungen von vulkanisiertem Kautschuk gefällt werden, z. B. bei der Bromid. bestimmung des Kautschuks nach S. Axeirod ${ }^{13}$,

12) Immerhin sind hier Versuche nach dieser Richtung, 2. B. auf mikroskopischem Wege, geplant. 4) Gummi-Ztg. 21, 1229 (1907). 
so wäre es doch sehr unwahrscheinlich anzunehmen, dab genau das gleiche Adsorptionsgleichgewicht, wie es ursprünglich zwischen festem Kautschuk und Schwefel bestanden hatte, auch bestehen bleiben würde, trotzdem der Kautschuk vorher gelost und dann wieder ausgefällt wurde.

Endlich ist nicht einzusehen, warum der Kautschuk nicht imstande sein sollte, mit Schwefel und Schwefelchlor ür Verbindungen einzugehen, während andererseits wohldefinierte Verbindungen mit Brom. Chlor, Salzsäure, Ozon usw. mit Sicherheit bekannt sind.

Nach alledem erscheint es gerechtfertigt, zur Erklärung der Schwefel- und Schwefelchlorüraufnahme bei der Vulkanisation auch die Bildung bestimmter chemischer Verbindungen in Betracht zu ziehen. Andererseits muB aber auch, wie aus den Betrachtungen Wo. Ostwald's und den Untersuchungen B. Bysow's, sowie von F. W. Hinrichsen und E. Kindscher übereinstimmend folgt, die Adsorption wie bei allen Kolloiden eine wesentliche Rolle spielen. Der scheinbare Gegensatz zwischen den Ergebnissen der zuletzt genannten Arbeiten erklärt sich dann ohne weiteres aus der grundsảtzlichen Verschiedenheit der Versuchsbedingungen.

Die Auffassung, daß als Vorgänge, welche die Vulkanisationserscheinungen bedingen, $\mathrm{so}$ wohl Adsorption als auch chemische Unsetzung in Frage kommen, führt aber sogleich einen vielleicht nicht unwichtigen Schritt weiter, sobald man die Reaktions ges chwindigkeit berücksichtigt. Denn Ads orptionsgleichgewichte pflegen sich, wie namentlich $H$. Freundlich feststellen konnte, mit auBerordentlich grober Geschwindigkeit einzustellen, während chemische $U \mathrm{~m}$ setzungen nicht dissozilerter organischer Stoffe in der Regel lang sam verlaufen. Man könnte sich demnach den Verlauf der Reaktion so vorstellen, daß bei der Vulkanisation $2 u$. nächst rein oberflächliche Adsorption des vulkanisierenden Mittels erfolgt und in zweiter Linie sodann die chem ische Bindung der adsorbierten St offe vor sich geht. Die Geschwindigkeit der chemischen Umsetzung muB naturgemäß in hohem Grade von den äuBeren Versuchsbedingungen (Konzentration des Schwefels bezw. Schwefelchlorïres, Druck und besonders Temperatur) abhänget. Bei der in der Technik angewandten Temperatur der Hei\&vulkanisation $\left(130^{\circ}\right.$ und höher) dürfte die Geschwindigkeit der chemischen Reaktion zwischen Kautschuk und Schwefel so groß sein, daB innerhalb der angewandten $Z$ eit (z. B. von einer Stunde) schon merkliche Mengen Schwefel in chemische Bindung übergegangen sein können.

Wenn diese Anschauung richtig ist, so mub man erwarten, daß der zweite Teil der Reaktion, die chemische Bindung des Schwefels, nicht nur bei den in der Technik benutzten Temperaturen der HeiBvulkanisation, sondern bei jeder beliebigen Temperatur stattfinden muB. Nur wird naturgema die Reaktionsgeschwindigkeit um so kleiner sein, je niedriger die angewandte Temperatur ist. Macht man die Annahme, dab der freie Schwefel dem nur adsorbi e r t e n Anteile entspricht - da Adsorptionen nach dem früher Gesagten umkehrbar sind, mub ja der nur adsorbierte Schwefel bei erschöpfender Behandlung mit einem Lösungsmittel (Azeton) dem Kautschuk vollständig entzogen werden können -- so müBte sich chemisch die genannte Reaktion dadurch nachweisen lassen, daß die Menge des freien Schwefels mit der Zeit ab*, die des gebundenen Schwefels zunähme.

Tatsächlich sind nun Vorgänge bekannt, welche auf eine solche Verschiebung des ursprünglichen Gleichgewichtes mit der Zeit zugunsten des gebundenen Schwefels hindeuten. Es handelt sich hierbei um die jedem Kautschuktechniker bekannten Erscheinungen der Nachvulkanisation.

Im "Königl. Materialprüfungsamte " liegen die ersten experimentellen Untersuchungen nach dieser Richtung schon ziemlich weit zurück. Den Ansto 6 gaben die bereits im vorigen Jahre an dieser Stelle erwahnten Versuche, die auf Veranlassung von Herrn Geh. Ober-Reg.-Rat Martens angestellt waren und die Prüfung des Einflusses verschiedener Lagerungsbedingungen auf die mechanischen Eigenschaften von Kautschuk zum Ziele hatten. Hand in Hand mit den mechanischen Prüfungen wurden damals auch chemische Untersuchungen ausgegeführt. Das Probematerial bestand aus einer Mischung von Para + Schwefel ohne weitere Zusatzstoffe. Die für die vorliegende Frage wichtigen Zahlen für den Gehalt an freiem und gebundenem Schwefel, die von R. Bernhardi-Grisson und $E$. Kindscher ermittelt wurden, sind in der folgenden $\mathrm{Ta}$ belle 1 zusammengestellt. Die Bestimmung des Gesamtschwefels erfolgte nach dem früher von mir beschriebenen Verfahren der elektrolytischen Oxydation in Salpetersäure ${ }^{14}$ ). Die Schwefel-

14) Chem.-Ztg. 33, 735 (1909). 
säure wurde gewichtsanalytisch oder meist auf titrimetischem Wege nach $P$. Raschigis) (Fällung als Benzidinsulfat und nachfolgende Titration mit Natronlauge) ermittelt. In gleicher Weise wurde auch bei den in der letzten Spalte befindlichen eingeklammerten Zahlen, bei denen der Gehalt an gebundenem Schwefel durch unmittelbare Untersuchung des mit Azeton erschöpfend ausgezogenen Materiales festgestellt wurde, verfahren. Dic Titration, die schnell

20) Zeitschr. f. angew. Chemie 1908, 617 und 818. S. auch v. K norre, Chem. Industrie 28, 2 (1905). auszuführen ist, lieferte sehr befriedigende $\mathrm{Er}$ gebnisse. Die Bestimmung des freien Schwefels erfolgte in dem nach zehnstündiger Extraktion im Soxhlet-Apparate erhaltenen Azetonauszuge durch Oxydation mit Salpetersäure, Eindampfen mit Salzsăure auf Zusatz von etwas Chlornatrium und nachberige Fallung als Bariumsulfat aus salzsaurer Losung ${ }^{16}$ ). Die Zahlen der Tabelle 1 stellen in der Regel das Mittel aus je zwei Vergleichsversuchen dar.

19) Vgl. P.W. Hinrichsen und K. Memmler, Der Keutschuk und seine Prufung (Leipzig 1910), 116.

Tabelle 1

EinfluB der Lagerung von Kautschuk auf den Gehalt an gebundenem Schwefel.

\begin{tabular}{|c|c|c|c|c|}
\hline $\begin{array}{l}\text { Nr. } \\
\text { des Versuchs }\end{array}$ & Art der Lagerung & $\begin{array}{c}\text { Gesamt- } \\
\text { schwefel } \\
\text { Pros. }\end{array}$ & $\begin{array}{c}\text { Freier } \\
\text { Schwefel } \\
\text { Proz. }\end{array}$ & $\begin{array}{l}\text { Gebundener } \\
\text { Schwefel } \\
\text { Proz. }\end{array}$ \\
\hline $\begin{array}{l}1 \\
2\end{array}$ & $\begin{array}{l}\text { Einlieferungszustand } \\
1 / 2 \text { Jahr } \\
\text { bei Zimmerwarme dunkel und feucht aufbewahrt }\end{array}$ & $\begin{array}{l}9,0 \\
8,9\end{array}$ & $\begin{array}{l}4,5 \\
4,3\end{array}$ & $\begin{array}{r}4,5 \\
4,6 \\
(4,6)\end{array}$ \\
\hline 3 & $\begin{array}{l}1,2 \text { Jahr } \\
\text { bei Zimmerwärme dunkel und trocken aufbewahrt }\end{array}$ & 9,0 & 4,1 & $\begin{array}{c}4.9 \\
(4,7)\end{array}$ \\
\hline $\begin{array}{l}4 \\
5\end{array}$ & 1/2 Jahr auf dem Dache des Amtes aufbewahrt & 8,4 & 2,3 & 6,1 \\
\hline 6 & $\begin{array}{c}\text { bei etwa } 70^{\circ} \mathrm{C} \text { dunkel und feucht aufbewahrt } \\
1 / 2 \text { Jahr }\end{array}$ & 8,5 & 2,0 & $\mathbf{6 , 5}$ \\
\hline & bei etwa $70^{\circ} \mathrm{C}$ dunkel und trocken aufbewahrt & & $\sqrt{1,1}$ & ס , \\
\hline
\end{tabular}

Aus den Zahlen der Tabelle 1 folgt, daB bei Aufbewahrung des Kautschuks bei etwa $70^{\circ}$ (Versuch 5 und 6) und sogar bei gewöhnlicher Temperatur im Lichte (Versuch 4) innerhalb eines halben Jahres eine merkliche Abnahme des freien und Erhöhung des gebundenen Schwefels erfolgt ist. Die geringeren Betrüge an Gesamtschwefel bei den zuletzt angeführten Versuchen sind wohl durch Ausblühen und spätere mechanische Entfernung des Schwefels zu erklären.

Eine weitere Versuchsreihe, die bei $8090^{\circ}$ unter Anwendung eines anderen ebenfalls nur aus Kautschuk und Schwefel zusammengesetzten Materials durchgeführt wurde, zeigte, daB bei dieser etwas erhohteren Temperatur schon im Verlaufe weniger Tage die gleiche Erscheinung beobachtet werden konnte. Die Ausführung der Analysen durch R. Bernhardi.Grisson und $E$. K inds cher erfolgte auf gleiche Weise wie bei den vorher beschriebenen Versuchen. Folgende Zahlen wurden erhalten (s. Tab. 2).

Die in eckigen Klammern befindlichen Zahlen (Versuch 2), die nur einmal bestimmt werden konnten, fallen aus dem Rahmen der übrigen so weit heraus, daB wohl ein Versuchsfehler
Tabelle 2

Zunahme des gebundenen Schwefels bei trockener dunkler Lagerung des Kautschuks bei $80-90^{\circ} \mathrm{C}$.

\begin{tabular}{c|c|c|c|c}
\hline $\begin{array}{c}\text { Nr. des } \\
\text { Versuchs }\end{array}$ & $\begin{array}{c}\text { Dauer des } \\
\text { Lagerns } \\
\text { rage }\end{array}$ & $\begin{array}{c}\text { Gesamt- } \\
\text { schwefel } \\
\text { Proz. }\end{array}$ & $\begin{array}{c}\text { Freier } \\
\text { Schwefel } \\
\text { Proz. }\end{array}$ & $\begin{array}{c}\text { Gebund. } \\
\text { Schwwefel } \\
\text { Proz. }\end{array}$ \\
\hline 1 & 0 & 6,4 & 4,7 & $1,7(1,7)$ \\
2 & 1 & {$[5,91$} & 4,7 & {$[1,2]$} \\
3 & 2 & 6,6 & 4,5 & 2,1 \\
4 & 5 & 6,4 & 4,6 & 1,8 \\
5 & 10 & 6,7 & 3,5 & 3,2 \\
6 & 20 & 6,4 & 1,9 & 4,5
\end{tabular}

anzunehmen ist. Aus den übrigen Zahlen folgt in Uebereinstimmung mit Tabelle 1, dab auch bei Temperaturen, die merklich unterhalb der in der Technik für die Heillvulkanisation benutzten liegen, bereits eine wesentliche Zunahme des gebundenen Schwefels erfolgt.

Diese Beobachtungen geben ein Mittel an die Hand, experimentell noch naher in die Kinetik der Vulkanisationsvorgänge einzudringen. (jeht man nämlich von $u n$. vulkanisierten Mischungen von Kautschuk und Schwefel aus, so ist man durch Wahl geeigneter Temperaturen imstande, die Schwefel- 
aufnahme in chemischer Bindung gerade mit solcher Geschwindigkeit vor sich gehen zu lassen, $\mathrm{daB}$ man sie bequem messend verfolgen kann. Derartige Versuche haben, abgesehen von ihrem theoretischen Interesse, auch nicht zu unterschätzende praktische Bedeutung, da sie gestatten, die Vulkanisierfähigkeit verschiedener Kautschuksorten $\mathbf{z a h l e n m a ̈ B i g ~ m i t e i n a n d e r ~}$ zu vergleichen. Besonders wichtig erscheint ferner die Tatsache, daß hier ein Weg gegeben ist, um den EinfluB beliebiger Arten von Füllstoffen aufdie Geschwindigkeit der Vulkanisation ohne weiteres experimentell zu ermitteln. Hier ist noch eine große Lücke auszufüllen. Es ist bekannt, daß gewisse Zusatzstoffe, z. B. Bleioxyd, die Vulkanisation beschleunigen, andere unter Umständen verzögern können. Im Falle des Bleioxyds ist durch die schöne, kürzlich erschienene Arbeit $\mathrm{S}$ e i d l's ${ }^{17}$ ) sogar schon mit einiger Sicherheit nachgewiesen, worauf die begünstigende Wirkung des Bleioxyds zurückzuführen ist. Allgemeine Gesetzmäbigkeiten sind aber zurzeit noch nicht bekannt, soweit sie nicht in der Technik durch langjährige Erfahrung festgestellt sind und naturgemäß geheim gehalten werden. Nachdem einige Vorversuche mit Mischungen von Kautschuk und Schwefel, sowie von Kautschuk, Schwefel und Bleioxyd gezeigt haben, daß die planmäBige Verfolgung dieses Problems schon bei 50,70 und $90^{\circ} \mathrm{C}$ keine Schwierigkeiten macht, sind zurzeit im Königl. Materialprüfungsamte größere Versuchsreihen im Gange, welche die Erkenntnis des Einflusses zunächst der wichtigsten anorganischen Fülstoffe zum Ziele haben. Die auf dem beschriebenen Wege erhaltenen Ergebnisse, über die ich bald im $\mathrm{Zu}$ sammenhange berichten zu können hoffe, haben den Vorzug, dat sie sich unmittelbar an die in der Technik benutzten Verfahren der Vulkanisation anlehnen.

17) Gummi-Ztg. 25, 710, 748 (1911); siehe ferner W. Esch, Gummimarkt 4. 71 (1910), sowie W. Esch and $M$. Auerbach. ib. 5,123 (1911).
Vielleicht nicht ohne praktische Bedeutung dürften ferner weitere Versuchsreihen sein, die ebenfalls hier in Vorbereitung sind und den Einfluß höherer Drucke auf die Vulkanisation zum Ziele haben. Die zurzeit auf dem Gebiete des Kautschuks herrschende überaus rege experimentelle und literarische Tätigkeit veranlabit mich, schon jetzt die Richtlinien für unsere Arbeiten bekannt zu geben.

Neben diesen Versuchen, die in erster Linie das Problem der Heißvulkanisation beleuchten sollen, sind wir auch mit der weiteren Durchprüfung der Theorie der Kaltvulkanisation in der schon früher von uns eingeschlagenen Richtung beschäftigt. Die Veranlassung hierzu boten vornehmlich einige Beobachtungen, welche zeigten, dabs das von uns bei den irüheren Versuchen angewandte Chlorbestimmungsverfahren unter Umständen merkliche Fehler aufweisen kann. Ueber diese Versuche wird ausführlicher an anderer Stelle berichtet. Gewisse neuere Beobachtungen scheinen dafür zu sprechen, $\mathrm{daB}$ die in unserer vorläufigen Mitreilung angenommene Formel der chemischen Verbindung zwischen Kautschuk und Schwefelchlorür vielleicht nicht in allen Fällen zutrifft. Allerdings sind zu den neuen Versuchen andere Kautschuksorten als früher verwendet worden. Hierüber kann aber erst nach Ausführung umfassender Untersuchungen Sicheres ausgesagt werden. An dieser Stelle sei nur noch erwähnt, daB zum Zwecke der Vereinfachung der früher beschriebenen umständlichenVersuchsanordnung zur Bestimmung des Schwefelchlorïrs von $R$. Bernhardi-Grisson ein einfaches titrimetrisches Verfahren ausgearbeitet worden ist, das auf der Zersetzung der benzolischen Schwefelchlorürlösung durch alkoholische Natronlauge in Gegenwart von Wasserstoffsuperoxyd und nachfolgender Titration des Chlors mit Silbernitrat und Rhodanammonium beruht. Auch über diese Versuche wird später im Zusammenhange berichtet werden.

\section{Zur Kenntnis der Kaltvulkanisation.}

\section{Bemerkungen zu der Veröffentlichung von B. Bysow.}

Von F. Willy Hinrichsen, Groh-Lichterfelde.

(Eingeg. ans 15. April 19:1)

In Heft 4 dieser Zeitschrift kommt B. By sow ${ }^{1}$ ) auf Grund eigener Versuche über den EinfluB von Feuchtigkeit auf die Menge des bei der

1) Koll.-Zeitschr. 8. 209 (1911).
Kaltvulkanisation gebundenen Schwefels zu dem Schlusse, daR im Gegensatz zu einer angeblich von mir geäußerten Ansicht bei Anwendung feuchter Reagenzien weniger Schwefel gebunden wird. als in trockenem Zustand. Eine Ansicht 